\title{
Intracellular Staphylococcus aureus triggers pyroptosis and contributes to inhibition of healing due to perforin-2 suppression
}

\author{
Irena Pastar, ${ }^{1}$ Andrew P. Sawaya, ${ }^{1}$ Jelena Marjanovic, ${ }^{1}$ Jamie L. Burgess, ${ }^{1}$ Natasa Strbo, ${ }^{2}$ Katelyn E. Rivas, ${ }^{2}$ \\ Tongyu C. Wikramanayake, ${ }^{1}$ Cheyanne R. Head, ${ }^{1}$ Rivka C. Stone, ${ }^{1}$ Ivan Jozic, ${ }^{1}$ Olivera Stojadinovic, ${ }^{1}$ Eran Y. Kornfeld, ${ }^{1}$ \\ Robert S. Kirsner, ${ }^{1,3}$ Hadar Lev-Tov, ${ }^{1,3}$ and Marjana Tomic-Canic ${ }^{1}$
}

${ }^{1}$ Wound Healing and Regenerative Medicine Research Program, Dr. Phillip Frost Department of Dermatology and Cutaneous Surgery, ${ }^{2}$ Department of Microbiology and Immunology, and ${ }^{3}$ Wound Healing Clinical Research Program, University of Miami Miller School of Medicine, Miami, Florida, USA.

\begin{abstract}
Impaired wound healing associated with recurrent Staphylococcus aureus infection and unresolved inflammation are hallmarks of nonhealing diabetic foot ulcers (DFUs). Perforin-2, an innate immunity molecule against intracellular bacteria, limits cutaneous infection and dissemination of $S$. aureus in mice. Here, we report the intracellular accumulation of $S$. aureus in the epidermis of DFUs with no clinical signs of infection due to marked suppression of perforin-2. S. aureus residing within the epidermis of DFUs triggers AIM2 inflammasome activation and pyroptosis. These findings were corroborated in mice lacking perforin-2. The effects of pyroptosis on DFU clinical outcomes were further elucidated in a 4-week longitudinal clinical study in patients with DFUs receiving standard care. Increased AIM2 inflammasome and ASC-pyroptosome coupled with induction of IL-1 $\beta$ were found in nonhealing DFUs compared with healing DFUs. Our findings revealed that perforin-2 suppression, intracellular S. aureus accumulation, and associated induction of pyroptosis contribute to healing inhibition and prolonged inflammation in patients with DFUs.
\end{abstract}

\section{Introduction}

Diabetic foot ulcers (DFUs) are a serious complication of diabetes mellitus and a frequent cause of morbidity, including infection and lower leg amputation (1-4). More than half of DFUs are clinically infected and are especially associated with poor outcomes $(5,6)$. Staphylococcus aureus has been identified as the most prevalent pathogen in DFUs (7-12). DFUs are also characterized by unresolved inflammation preventing progression to healing $(8,13-17)$. Despite the high rate of recurrent $S$. aureus colonization and infection, the mechanisms by which this pathogen contributes to prolonged inflammation and inhibition of healing in DFUs are not yet fully understood.

Perforin-2 (P-2) is a membrane attack complex perforin (MACPF) domain containing protein that is associated with broad spectrum bactericidal activity against intracellular bacteria (1823). P-2 is encoded by the Mpeg1 gene and was initially identified based on homology to the MACPF domain of perforin 1, the cytolytic protein expressed by NK cells and cytotoxic T cells (CTLs) $\mathrm{i}(24)$. We have shown previously that constitutive expression of P-2 in the epidermis and primary human keratinocytes plays important

Authorship note: IP and APS contributed equally to this work.

Conflict of interest: RSK is a consultant for Mölnlycke and has pending patent application US 2020/0100711 A1. HLT is a consultant for Next Science Inc. and Pfizer Inc. MTC's research is supported by Organogenesis Inc.

Copyright: (c) 2021, American Society for Clinical Investigation.

Submitted: September 23, 2019; Accepted: October 27, 2021;

Published: December 15, 2021

Reference information: / Clin Invest. 2021;131(24):e133727.

https://doi.org/10.1172/JCl133727. roles in protection from infection and invasion by $S$. aureus (20, 23). Mpeg1 $1^{-/-}$mice are unable to limit the proliferation and dissemination of bacteria and succumb to bacteremia after epicutaneous exposure to $S$. aureus at a dose nonlethal to WT littermates (20). Recent studies show that $S$. aureus wound infection results in striking suppression of P-2 and inhibition of healing in ex vivo human acute wound healing (23). However, despite the importance of P-2 in cutaneous bacterial defense, the role of P-2 in the pathological chronicity of DFUs remains unknown.

Pyroptosis is a caspase- 1 dependent, highly inflammatory form of cell death that results in cell lysis and release of its proinflammatory contents (25-29). Execution of pyroptosis is an inflammasome-mediated process induced by the presence of intracellular pathogens (27, 30-32). Absent in melanoma 2 (AIM2) was identified as an inflammasome-forming protein in response to cytosolic dsDNA originating from intracellular pathogens (32-35). Activation of AIM2 leads to the oligomerization of the inflammasome adaptor protein apoptosis-associated speck-like protein containing a CARD domain (ASC) into a large supramolecular complex termed the pyroptosome (28, 32, 34-36). Pyroptosome assembly induces cleavage and activation of procaspase-1, which in turn activates caspase-1, resulting in proteolytic processing of the pore-forming protein, gasdermin $\mathrm{D}$. This cascade triggers formation of pores in the plasma membrane, resulting in cell lysis and release of intracellular contents and inducing an inflammatory response $(27,37,38)$. Recent studies have shown that inflammasome components are expressed in several skin cell types, including keratinocytes $(25,33$, $36,39,40$ ); however, the role of pyroptosis and its relevance to healing inhibition in DFUs have not been reported. 
A

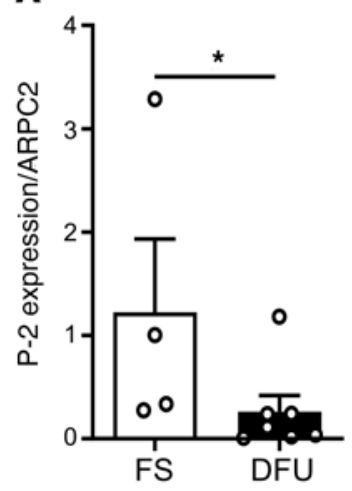

D
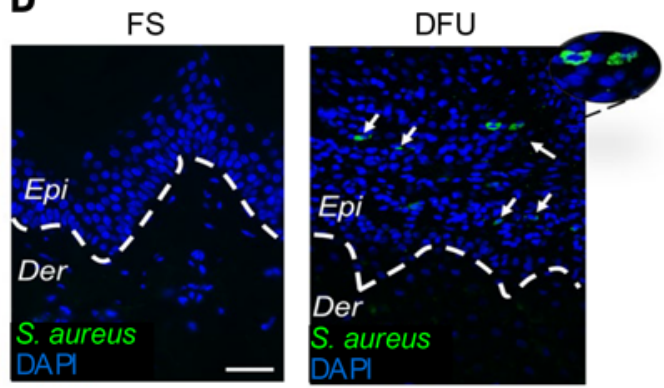

E

B
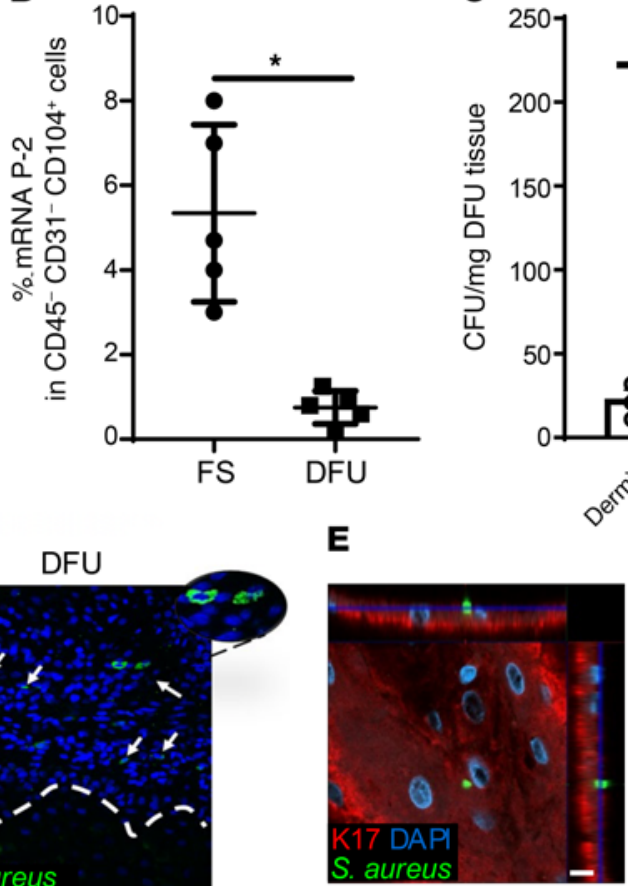

Figure 1. Suppression of $\mathbf{P}-\mathbf{2}$ in the epidermis of DFUs allows intracellular accumulation of $\mathbf{S}$. aureus. (A) Relative expression of P-2 was assessed by qPCR normalized to Arpc 2 in control foot skin (FS; $n=5$ biological replicates, 15 technical replicates) and diabetic foot ulcers (DFUs; $n=6$ biological replicates, 18 technical replicates). (B) P-2 mRNA levels in control and DFU keratinocytes quantified using FISH-Flow. Live CD45$\mathrm{CD} 31^{-}$, and CD104+ cells were analyzed for P-2 RNA expression within epidermal keratinocytes ( $n=5$ biological replicates). (C) Quantification of intracellular bacterial load in the epidermis and dermis per milligram of tissue ( $n=5$ biological replicates, 15 technical replicates). (D) Immunofluorescence staining of FS and DFU with S. aureus-specific antibody (green). Arrows indicate presence of intracellular S. aureus. DAPI = nuclei; dashed line demarcates epidermal-dermal boundary; Epi, epidermis; Der, dermis; scale bar: $50 \mu \mathrm{m}$. (E) Confocal imaging of DFU tissue stained with keratin 17 (K17; red) and S. aureus-specific antibody (green). DAPI (blue) $=$ nuclei; images taken at $63 \times$ magnification, scale bar: $10 \mu \mathrm{m} .{ }^{*} P<0.05$ (A and $\left.\mathbf{B}\right) ;{ }^{* *} P<0.01$ (C).

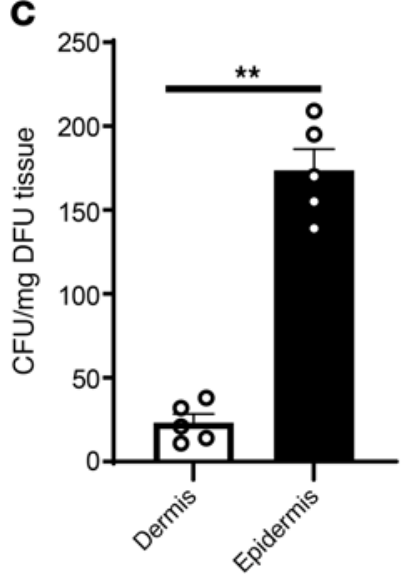

23) leads to the premise that human cutaneous conditions with P-2 suppression or insufficiency may be susceptible to $S$. aureus intracellular infection (41). To test this, we evaluated the expression of P-2 in chronic DFUs by real-time PCR (qPCR) and found suppression of $\mathrm{P}-2$ in the ulcer tissue compared with location-matched controls (Figure 1A). Next, we analyzed $\mathrm{P}-2$ expression in DFUs on a single-cell level by FISH-Flow (23) and found marked suppression of P-2 expression in $\mathrm{CD}_{104^{+}}$basal DFU keratinocytes (Figure 1B). Previously, we showed that $S$. aureus suppresses P-2 in keratinocytes during wound infection of human skin (23). Thus, we postulate that $\mathrm{P}-2$ suppression in DFUs results in accumulation of intracellular $S$. aureus. To evaluate the presence of the intracellular pathogens in DFUs, we isolated epidermis and dermis from the ulcer tissue in the presence of gentamicin, lysozyme, and lysostaphin in order to remove all extracellular bacteria and subjected cell suspension to hypotonic lysis, releasing bacteria from skin cells. A higher intracellular load of S. aureus was found in the DFU epidermis compared with the dermis (Figure 1C), whereas it was absent in location-matched controls. Presence of intracellular $S$. aureus in the epidermis of DFUs was confirmed by immunohistochemistry, costaining with epi-

We investigated the role of P-2 in the pathophysiology of chronic DFUs in the absence of clinical signs of infection, which are characterized by unresolved inflammation (17). We found striking suppression of P-2 in DFU keratinocytes that allowed intracellular S. aureus accumulation in the epidermis. We showed induction of ASC pyroptosome assembly and AIM2 activation by intracellular S. aureus in primary human keratinocytes and DFU tissue, which was corroborated in vivo in $\mathrm{P}-2-\mathrm{KO}\left(\mathrm{Mpeg}^{-}\right)$mice. Furthermore, induction of pyroptosis in DFUs was associated with activation of gasdermin D and increased levels of IL-1 $\beta$, which contributed to prolonged inflammation. AIM2 inflammasome activation and increased levels of pyroptosome components demonstrated correlation with the nonhealing outcome of DFUs. We conclude that $S$. aureus, by suppressing $\mathrm{P}-2$, persists within DFU keratinocytes and contributes to prolonged inflammation and inhibition of healing by induction of pyroptosis.

\section{Results}

$P$-2 is suppressed in the epidermis of DFUs, allowing intracellular accumulation of S. aureus. Systemic dissemination of S. aureus due to epicutaneous infection in the P-2-KO mice coupled with suppression of P-2 in a human ex vivo wound model infected with $S$. aureus (22, dermal marker keratin 17 (K17), and confocal microscopy (Figure D and E, and Supplemental Video 1; supplemental material available online with this article; https://doi.org/10.1172/JCI133727DS1).

Intracellular $S$. aureus induces pyroptosis in DFU tissue and in human keratinocytes. To determine the potential presence of pyroptosis in DFUs due to intracellular S. aureus, we analyzed the histology of tissue samples obtained from the ulcer edge, where pathologically stalled keratinocytes accumulate. Location-matched nonulcerated plantar diabetic foot skin (FS) served as the control. Morphological analyses revealed a thick cornified layer, characteristic of plantar skin in all samples (Figure 2A). However, hole-like structures indicative of pyroptosis were found only in DFU tissue and were absent in control samples (Figure 2A). A marker for pyroptosis is the assembly of the pyroptosome, a supramolecular assembly of ASC oligomers. In order to confirm the presence of pyroptosis in tissue samples, chemical cross-linking of the ASC pyroptosome was performed followed by assessment of oligomers by Western blot. In line with histology assessments, FS controls showed absence, whereas DFU tissue samples showed induction of pyroptosome assembly, as indicated by the dimer and higher-ordered oligomer structures of ASC (Figure 2B). To rule out the possibility of cell death by apoptosis, we assessed an 
A
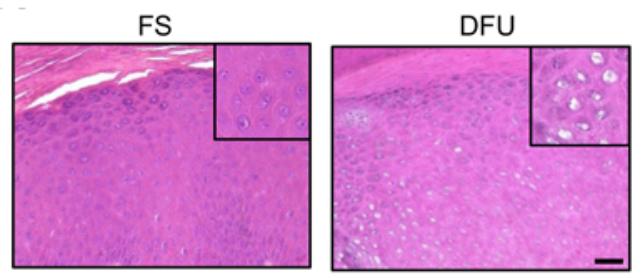

B

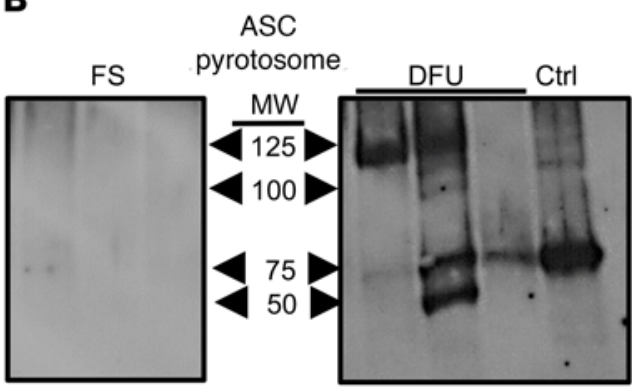

C

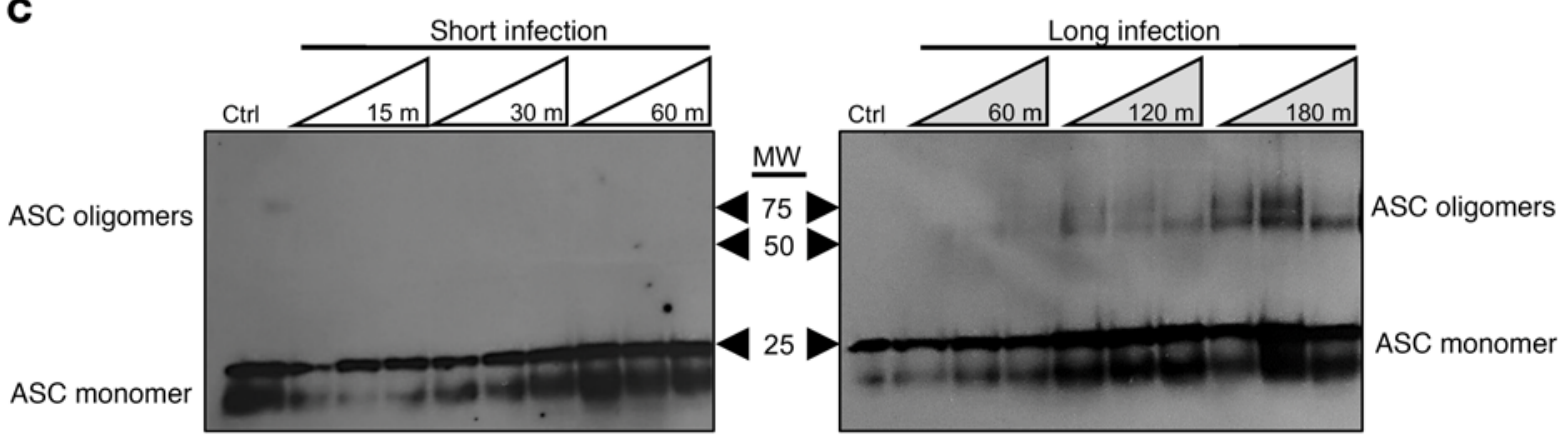

D
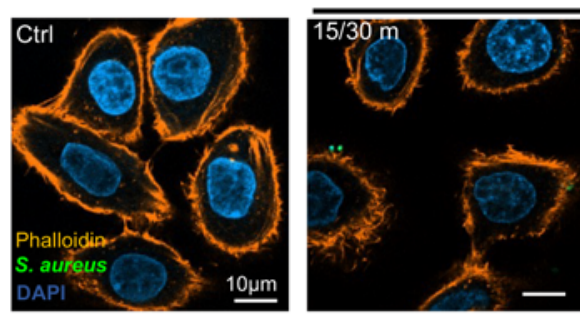

Short infection

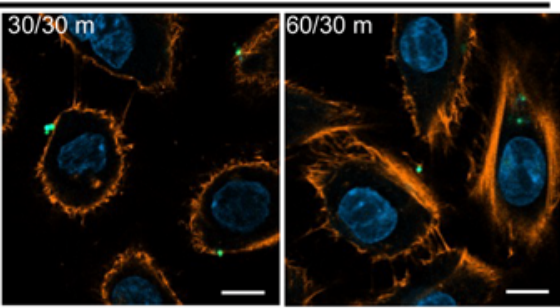

Long infection
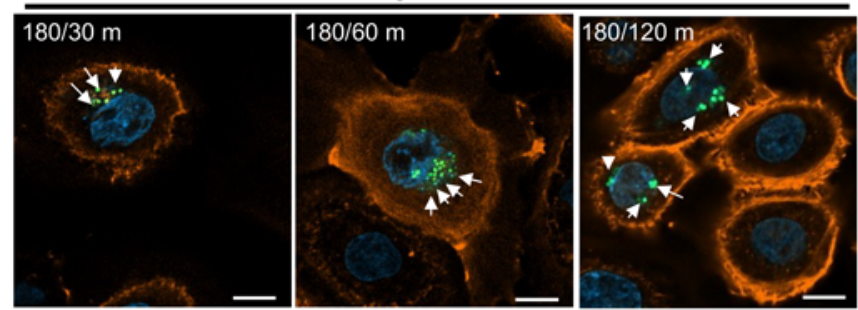

Figure 2. Intracellular presence of S. aureus induces ASC pyroptosome assembly in DFUs. (A) H\&E staining of healthy foot skin (FS) and diabetic foot ulcers (DFUs) revealing presence of hole-like structures indicative of cell lysis, suggesting presence of pyroptosis. (B) Western blot of ASC pyroptosome showing ASC oligomerization in DFUs ( $n=3$ per group), demonstrating induction of pyroptosis in DFUs; Ctrl= human keratinocytes treated with poly dA/ $\mathrm{dT}$ to induce pyroptosis. (C) Western blot of ASC pyroptosome demonstrated lack of ASC oligomerization during shorter infection allowing for bacterial adhesion (left blot) and induction of pyroptosis during longer infection allowing for MRSA internalization (right blot). Primary human keratinocytes were infected with MRSA at MOI of 100 for 15, 30, and 60 minutes; washed with PBS; and collected at 15-minute time increments (white triangles); or infected for 60, 120, and 180 minutes; washed; and collected at 30, 60, and 120 minutes (gray triangles) to allow bacteria to be internalized (20). Ctrl = uninfected cells. (D) Representative confocal imaging, corresponding to Western blots, of primary human keratinocytes infected with MRSA USA300 (green); DAPI (blue) = nuclei; rhodamine-conjugated phalloidin (orange) used to visualize actin; Ctrl = uninfected cells. Upper panel shows MRSA attached to keratinocytes during shorter infection time; bottom panel confirms intracellular localization of MRSA (white arrows); scale bar: $10 \mu \mathrm{m}$. Results depict representative experiment from 3 biological replicates.

apoptotic marker, caspase-3 activation, in DFUs by immunoblotting. In both control and DFU samples, only the pro-form of caspase-3 (39 $\mathrm{kDa}$ ) was found, whereas its cleaved, activated form (19 kDa) was not detected (Supplemental Figure 1), confirming absence of apoptotic cells in DFUs. In line with this finding, we did not detect apoptotic DNA fragmentation in DFU tissue (Supplemental Figure 2).
Next, we assessed whether the presence of intracellular bacteria induces pyroptosis in primary human epidermal keratinocytes. To test this, we used an $S$. aureus strain isolated from a DFU (8). Infected and uninfected human epidermal keratinocytes were either treated by lysostaphin to eliminate extracellular bacteria or left untreated $(20,23)$ and collected at multiple time points upon 
infection; then, pyroptosis was assessed by chemical cross-linking of ASC (Figure 2C). The presence of intracellular S. aureus was confirmed by immunostaining and confocal microscopy corresponding to induction of ASC pyroptosome, while bacteria attached to the cell wall did not (Figure 2D). We confirmed induction of pyroptosome assembly in human epidermal keratinocytes infected with intracellular $S$. aureus, as indicated by the oligomer structures of ASC in human epidermal keratinocytes (Figure 2, C and D).

Induction of pyroptosis is associated with increased IL-1 $\beta$. Activation of AIM2 inflammasome has been shown to induce the oligomerization of ASC into the pyroptosome, which in turn activates procaspase-1, leading to cleavage and activation of the pore-forming protein gasdermin $\mathrm{D}$ and pro-IL-1 $\beta$, resulting in inflammatory cell lysis. We evaluated AIM2 levels in DFUs by immunoperoxidase staining (Figure 3A). DFU samples showed induction of AIM2, in contrast to control FS that showed absence of AIM2 (Figure 3A). Further, we assessed AIM2 inflammasome activation by Western blot. We confirmed AIM2 induction specifically in DFUs, whereas it was absent in control FS (Figure 3B). To corroborate findings from human tissue, we performed transcutaneous infection in P-2-KO and WT mice. Infection with methicillin-resistant $S$. aureus (MRSA) induced AIM2 in the P-2-KO skin compared with WT skin in vivo and in vitro (Figure 3, C and D, and Supplemental Figure 3). Accumulation of intracellular MRSA in the keratinocytes of P-2-KO was evident, whereas it was absent in the WT skin, as confirmed by confocal imaging (Figure 3E).

Next, we evaluated the downstream effector of AIM2, caspase-1, in DFU samples. The caspase-1-cleaved activated form (10 kDa) was selectively found in DFU samples, and not in control location-matched FS (Figure 3F). To further confirm the induction of pyroptosis in DFU samples, we assessed the main effector of pyroptosis, gasdermin D (Figure 3G). We found cleavage and activation of gasdermin D to be present only in DFUs, whereas it was absent in control FS (Figure 3G). To assess expression levels of genes regulated by induction of pyroptosis, we utilized a DFU gene expression data set (8). We found a deregulated set of genes known to be involved in pyroptosis, including IL-1 $\beta$, gasdermin D, and AIM2 (Figure 3H). To confirm the microarray data and test whether induction of pyroptosis in DFU is associated with increased inflammation, IL-1 $\beta$ levels were assessed by ELISA in DFU tissue and FS controls. We found increased levels of IL-1 $\beta$ in DFUs (Figure 3I), suggesting that induction of pyroptosis induces IL-1 $\beta$, a proinflammatory cytokine, thus contributing to the persistent inflammation seen in DFUs. Furthermore, increased levels of IL-1 $\beta$ were confirmed in the skin of P-2-KO mice after infection with MRSA when compared with control WT skin (Figure 3J).

Pyroptosis correlates with the healing outcomes of DFUs. Next, we performed a 4-week longitudinal clinical study to investigate whether intracellular accumulation of $S$. aureus followed by induction of the AIM2 inflammasome and pyroptosis contributes to a nonhealing phenotype. We obtained tissue samples from a prospective set of patients at the initial visit (week 0, WO) and after 4 weeks of standard wound care (week 4, W4). The healing outcome was determined by a surrogate endpoint as a percentage reduction in wound size at W4 compared with WO (42). Patients were grouped in 2 categories: healers, in which wound reduction was greater than or equal to $50 \%$ and non-healers, in which wound reduction was less than 50\% (Supplemental Figure 4). Pyroptosis was assessed by chemical cross-linking of the ASC pyroptosome, and ASC oligomers were evaluated and quantified by Western blot. We found that pyroptosome assembly was induced in all DFU samples at WO. However, healers showed a decrease in pyroptosome assembly at $\mathrm{W} 4$, whereas pyroptosome assembly persisted or increased in nonhealing DFUs at W4 (Figure 4, A and B). In addition, we tested the correlation of the AIM2 inflammasome levels with the healing outcome of DFUs and found a similar pattern. All DFU tissue samples showed presence of AIM2 at WO (Figure 4C). Decrease of AIM2 at W4 compared with WO was found in healing DFUs, whereas AIM2 was increased at W4 in nonhealing DFUs (Figure 4, C and D). We then assessed whether gasdermin $\mathrm{D}$ activation correlated with healing in prospectively collected tissue and found cleaved and activated gasdermin D decreased at W4 compared with WO in healing DFUs, whereas gasdermin D was increased in nonhealing DFUs (Figure 4, E and F). Next, we assessed the levels of $S$. aureus in FFPE tissue from the healing and nonhealing DFUs by qPCR. DNA from the DFU FFPE samples was extracted and the presence of the $S$. aureus-specific thermonuclease gene (nuc) was quantified and normalized to total bacterial load by qPCR $(8,43)$. All DFU tissue samples were positive for $n u c$, confirming accumulation of $S$. aureus in the ulcer even without clinical signs of infection (Figure 4G); however, increased levels of $S$. aureus were detected in nonhealing DFU tissue (Figure 4G). Taken together, our results demonstrated that pyroptosome assembly and AIM2 inflammasome were induced in DFUs because of the accumulation of intracellular $S$. aureus, likely as a result of the suppression of innate immune molecule P-2 in the epidermis. Importantly, AIM2 inflammasome and ASC pyroptosome activation persisted in patients with nonhealing DFUs and correlated with higher levels of $S$. aureus.

\section{Discussion}

Here, we showed that $S$. aureus, by suppressing P-2, can survive and persist in keratinocytes of the DFU epidermis. Intracellular $S$. aureus triggered AIM2 inflammasome activation and ASC pyroptosome assembly and resulted in increased IL- $1 ß$ levels in tissue samples obtained from chronic ulcers of patients with diabetes who exhibited no clinical signs of infection (Figure 5). S. aureus internalization within epidermal keratinocytes was confirmed in the P-2-KO animals, resulting in subsequent activation of AIM2 and induction of IL-1 $\beta$. Furthermore, when correlated with ulcer healing, we found persistence or increase of pyroptosis in nonhealing DFUs, whereas a decrease in pyroptosis was associated with healing. To our knowledge, these findings are the first to demonstrate the clinical relevance of P-2 suppression in DFUs in the context of intracellular $S$. aureus and unresolved inflammation triggered by pyroptosis. Suppression of indispensable antimicrobial effector P-2 in DFU tissue and accumulation of intracellular $S$. aureus in nonhealing ulcers even without clinical signs of infection may identify patients at higher risk of infection and further complications.

P-2 and its bactericidal role against $S$. aureus have only recently been described $(19,20)$, and this is the first report, to our knowledge, showing correlation between P-2 expression levels and the chronicity of DFUs. Downregulation of P-2 is a unique characteristic of DFUs, and our previously published data on gene expression profiles from human diabetic FS have shown lack of P-2 regulation in diabetic versus healthy nondiabetic FS (44). Intracellular S. aureus 
A

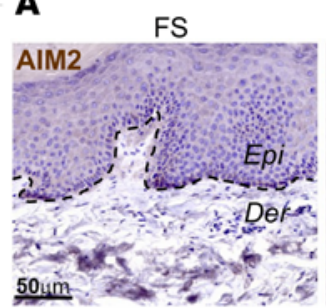

C

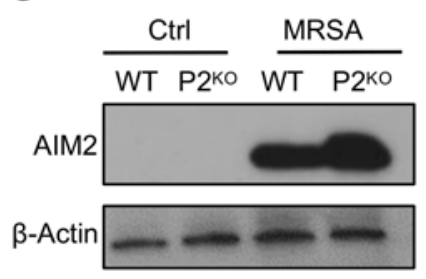

$\mathbf{F}$

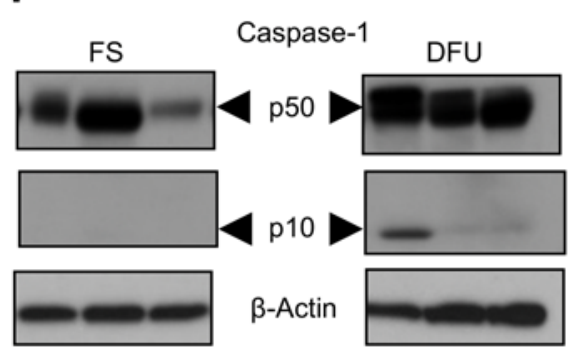

B

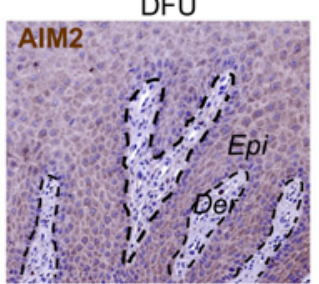

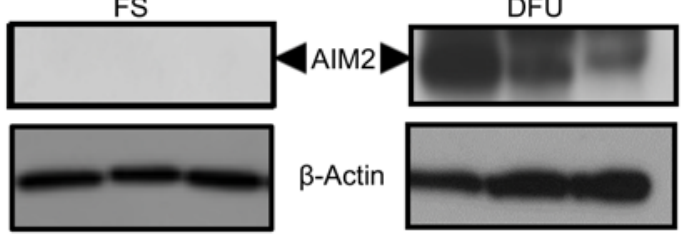

D

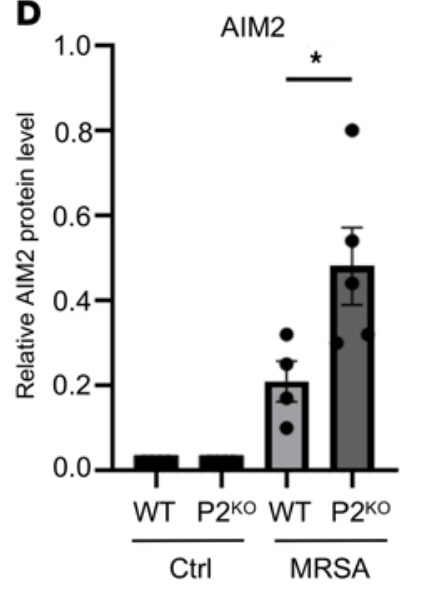

E

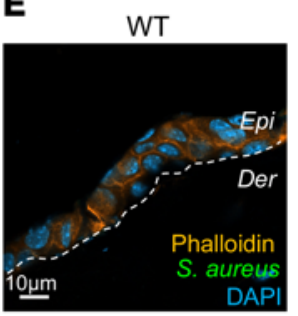

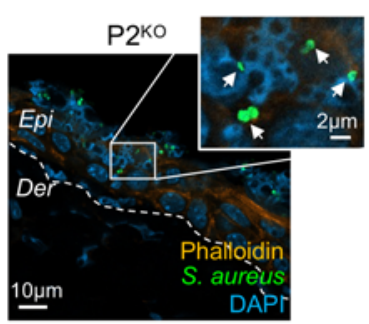

G

H

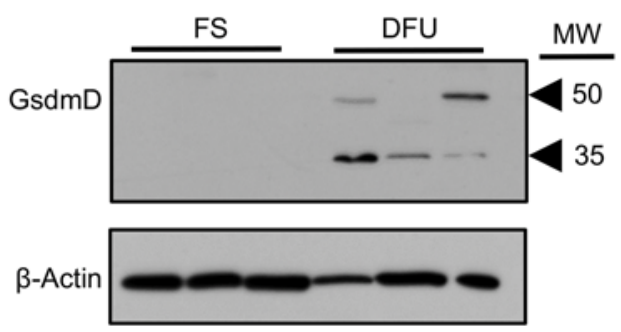

I

IL-1 $1 \beta$ DFU

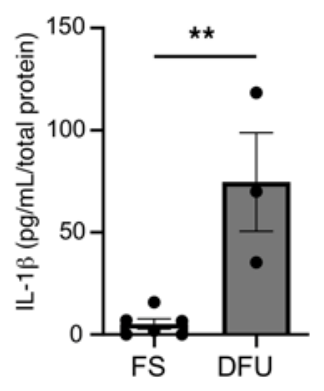

IL-1 $\beta$ murine

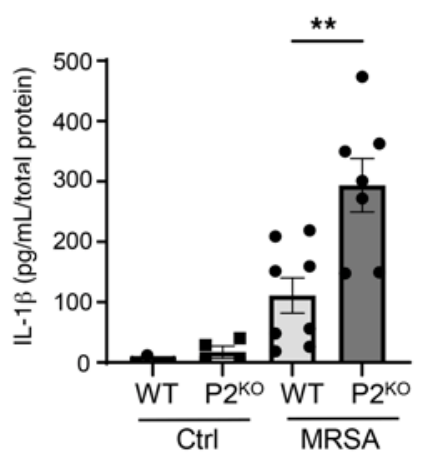

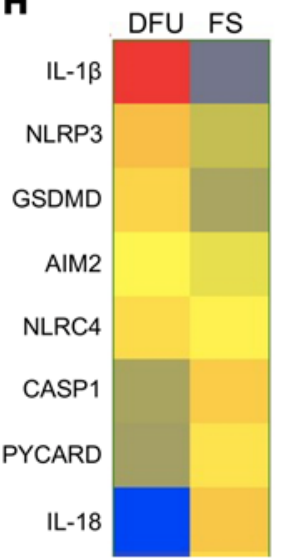

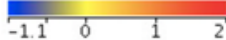

Figure 3. AIM2 inflammasome activation induces pyroptosis and increases IL-1ß activation in DFUs. (A) Immunoperoxidase staining of AIM2 in FS and DFUs. AIM2 was induced in DFUs compared with FS. Epi, epidermis; Der, dermis; dashed line demarcates epidermal/dermal boundary; scale bar: $50 \mu$ m. (B) Western blot of AIM2 in FS and DFUs ( $n=3$ per group). (C) Representative Western blot and quantification (D) of AIM2 levels in WT ( $n=4)$ and P-2-KO $(n=5)$ from murine skin in response to infection with MRSA USA3000 AH1726, validating that MRSA infection induced AIM2 with amplified induction in the skin from P-2-KO mice; ${ }^{*} P<0.05$. (E) Confocal imaging confirming intracellular MRSA in the epidermis of P-2-KO mice but not in the WT; Epi, epidermis; Der, dermis; dashed line demarcates epidermal/dermal boundary; arrows indicate intracellular MRSA; scale bar: $10 \mu \mathrm{m}$; inset scale bar: $2 \mu \mathrm{m}$. (F) Western blot caspase-1 in FS and DFUs ( $n=3$ per group). Caspase-1 is uniquely activated in DFUs compared with FS. (C) Western blot of gasdermin D (GsdmD; $n=3$ per group). Activation of GsdmD was found to be present in prospectively collected DFUs. (H) Gene signature of DFUs compared with control FS of a subset of genes involved in pyroptosis indicated strong induction of IL-1 $\beta$. (I) ELISA of IL-1 $\beta$ in FS and DFUs confirmed increased levels of IL-1 $\beta$ in DFUs ( $n=6$ biological replicates for FS, $n=3$ biological replicates for DFUs). Data are represented as mean \pm SEM and analyzed by unpaired 2-tailed $t$ test, ${ }^{* *} P<0.01$. (J) ELISA of IL-1 $\beta$ confirmed increased levels of IL-1 $\beta$ in the murine skin lacking P-2 after infection ( $n=4$ biological, 8 technical replicates for WT, $n=4$ biological, 7 technical replicates for P-2-KO). Data are represented as mean \pm SEM and analyzed by unpaired 2 -tailed $t$ test, ${ }^{* *} P<0.01$. 
A

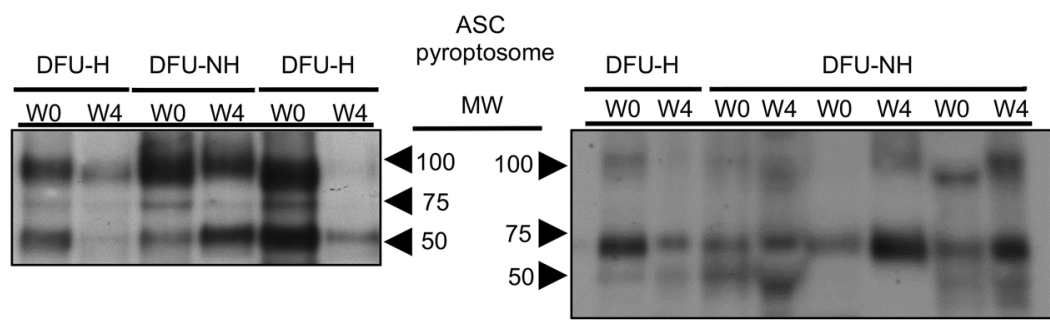

C

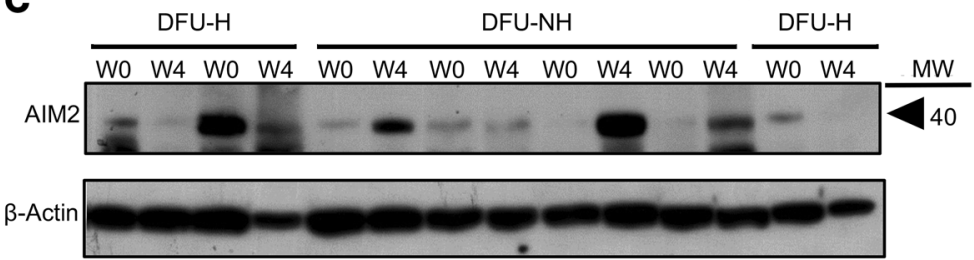

E

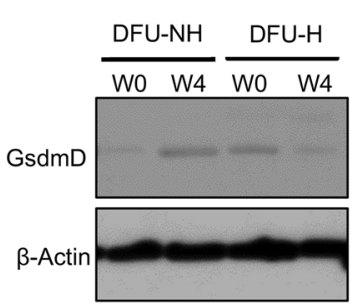

$\mathbf{F}$

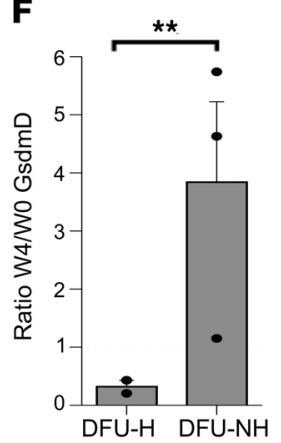

$\mathbf{G}$

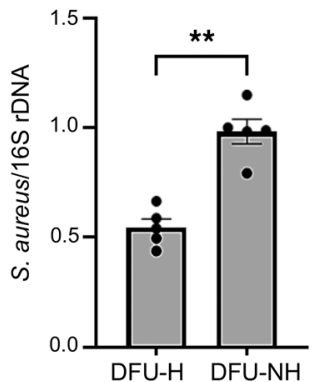

B

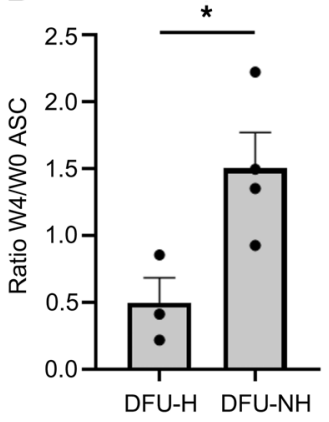

D

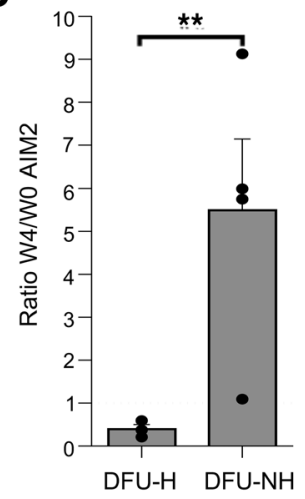

Figure 4. Pyroptosis correlates with the healing outcome of DFUs. (A) Western blot and corresponding quantification plot (B, the ratio of week $4 /$ week 0 (W4/WO) tissue levels for ASC) of ASC pyroptosome in healing (DFU-H; $n=3$ ) and nonhealing DFUs (DFU-NH; $n=4$ ); ${ }^{*} P<0.05$. (C) AIM2 Western blot and corresponding quantification plot (D; the ratio of W4/WO AIM2 tissue levels) in DFU-H $(n=3)$ and DFU-NH $(n=4)$. ASC pyroptosome and AIM2 levels decreased in DFU-H over time, whereas they persisted or increased in DFU-NH after 4 weeks (W4) of standard of care; ${ }^{* *} P<0.01$ ( $n=4$ per group). (E) Western blot of GsdmD in prospective DFU-H and DFU-NH. (F) Quantification of the ratio W4/WO levels for GsdmD in DFU-H and DFU-NH. Activation of GsdmD was found to persist or increase in DFU-NH at W4. ${ }^{* *} P<0.01$ ( $n=3$ per group). (C) Quantification of S. aureus levels by qPCR in tissue from DFU-H and DFU-NH. ${ }^{* *} P<0.01$ ( $n=5$ per group).

is of major clinical importance because it may provide antimicrobial resistance residing within this niche and contribute to recurrence of DFUs. Although certain virulence factors of $S$. aureus have been recently associated with healing inhibition of DFUs (7), their potential role in P-2 suppression and underlying mechanisms remain to be elucidated. The potent bactericidal activity of P-2 $(18,20,45)$ mandates suppression of P-2 expression in order for bacteria to establish even a temporary residence in the host's cells. Indeed, we recently demonstrated the ability of $S$. aureus to inhibit P-2 in human skin, resulting in the inhibition of healing, whereas overexpression of P-2 results in reduction of intracellular $S$. aureus in human keratinocytes (23). This leads to a conundrum: decrease of P-2 expression leads to invasion of intracellular $S$. aureus that further suppresses P-2 expression and, in turn, facilitates accumulation of intracellular $S$. aureus. In contrast to pathogenic $S$. aureus, commensal S. epidermidis is capable of inducing P-2 in keratinocytes and resident GDT cells, opening possibilities for therapeutic intervention (46).

Chronic unresolved inflammation is a major contributor to delayed healing in DFUs $(8,13,14,17,47,48)$. We have recently identified major transcriptional networks deregulated in DFUs that result in decreased neutrophil and macrophage recruitment and overall poorly controlled inflammatory response (17). Here, we demonstrated the role of inflammatory cell death, pyroptosis, triggered by suppression of P-2 and intracellular $S$. aureus, to inhibition of healing in DFUs. Cytosolic dsDNA originating from intracellular pathogens has been shown to trigger AIM2 inflammasome activation (32-35). We confirmed AIM2 inflammasome activation in DFUs and primary human keratinocytes due to intracellular $S$. aureus. Consequently, release of the host factors from microbialinduced pyroptotic cells may perpetuate AIM2 inflammasome activation and pyroptosis, promoting deregulation of the inflammatory response in DFUs, whereas the lack of functional neutrophils and macrophages may further affect bacterial clearance in DFUs (17). Release of intracellular $S$. aureus from pyroptotic cells may also promote ulcer colonization and perpetuate infection and its recurrence (Figure 5). Our data also demonstrated AIM2 correlation with healing outcomes, suggesting that AIM2 has an important role in modulating the inflammatory response in DFUs. We confirmed the role of $\mathrm{P}-2$ in AIM2 activation in the epidermis of P-2-KO animals. In P-2KO skin, AIM2 inflammasome levels were higher compared with 


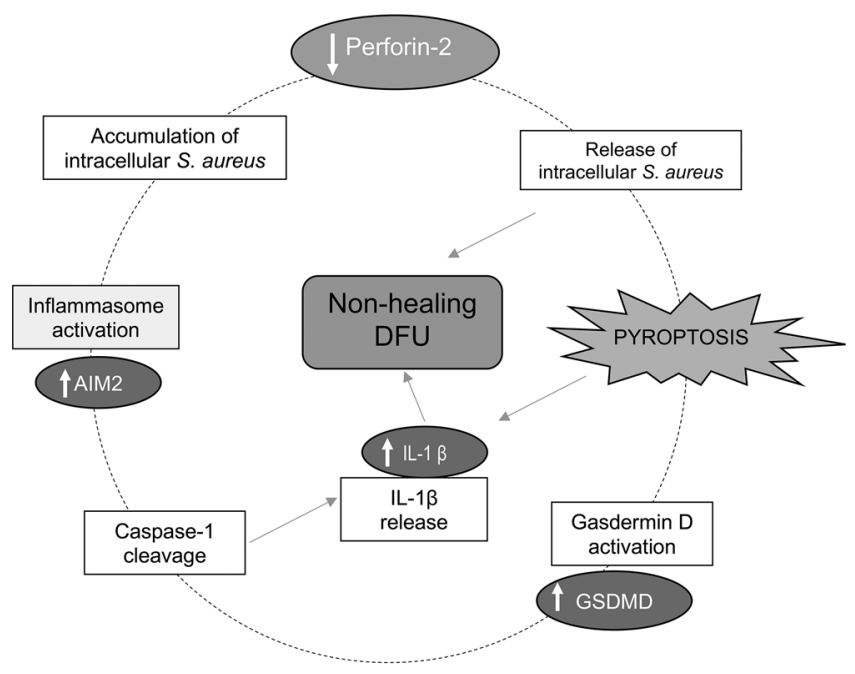

Figure 5. A current model of P-2/intracellular S. aureus-mediated induction of pyroptosis in DFUs. Accumulation of intracellular S. aureus in the DFU epidermis due to suppression of perforin-2 triggers AIM2 inflammasome activation, leading to caspase-1-mediated IL-1 $\beta$ activation and proteolytic processing of the pore-forming protein gasdermin $\mathrm{D}$. This cascade triggers formation of pores in the plasma membrane, resulting in pyroptosis and release of intracellular contents, including inflammatory mediators and accumulated intracellular S. aureus, contributing to chronic inflammation and inhibition of wound healing.

WT skin and correlated with accumulation of intracellular S. aureus and IL-1 $\beta$ after infection. This finding underscores the importance of $\mathrm{P}-2$ in skin barrier protection and maintenance.

Induction of ASC pyroptosome and subsequent activation of caspase-1 are in agreement with previous findings of increased levels of activated IL-1 $\beta$ in DFUs (48-51), suggesting that high levels of this proinflammatory cytokine in DFUs may be the result of increased pyroptosis. High levels of proinflammatory cytokines can have detrimental effects on wound healing because of subsequent induced expression of metalloproteases that excessively degrade the extracellular matrix and impair cell migration $(50,51)$. In addition, our laboratory has previously shown that IL-1 $\beta$ induces synthesis of epidermal cortisol, a potent wound healing inhibitor, resulting in impaired cell migration and wound closure (52-54). Therefore, it is possible that induction of pyroptosis in DFUs may promote excessive cortisol production, further contributing to unresolved inflammation and inhibition of healing.

We also found cleavage and activation of the caspase- 1 substrate, gasdermin D, to be uniquely present in DFUs. Gasdermin D has been shown to be the main effector of pyroptosis by acting as the pore-forming protein that results in cell lysis (37). Previous studies have implicated aberrant apoptosis to play a role in impaired wound healing in mice (55-57), but our data showed absence of apoptosis in DFUs and prevalence of pyroptosis. Caspase-3, a unique molecule found specifically in apoptosis but not in pyroptosis, was absent from DFUs, suggesting pyroptosis to be the predominant form of cell death in DFUs. Although the absence of apoptosis in DFUs was confirmed, the possibility of additional forms of cell death, such as necroptosis, remains to be validated. Furthermore, induction of gasdermin D in DFUs coupled with caspase-1 activation supports recent data documenting a gasdermin D requirement for caspase-1mediated pyroptosis (58).

Taken together, induction of pyroptosis due to intracellular $S$. aureus and its relationship with healing outcomes of DFUs identifies a pathway for persistent and recurrent bacterial colonization and prolonged inflammation in DFUs (Figure 5). The correlation between pyroptosis and healing outcomes of DFUs suggests that targeting the intracellular bacterial niche with the goal of inhibition of the pyroptotic pathway may promote healing. The identification of therapeutics that restore and/or increase P-2 levels may prevent S. aureus intracellular accumulation and allow the skin to successfully combat bacterial pathogens, prevent wound infection, and promote healing. In addition, evaluation of P-2 levels in DFU tissue in the future may serve as a biomarker that can predict infection.

\section{Methods}

Skin specimens. Eligible participants were adults aged 21 or older with type 2 diabetes mellitus and at least 1 clinically noninfected ulcer on the plantar aspect of their foot larger than $0.5 \mathrm{~cm}^{2}$. Inclusion and exclusion criteria are listed in Supplemental Data. Full-thickness skin specimens were obtained from wound edges of DFUs $(n=24$, mean age $\pm \mathrm{SD}=57 \pm 11.9,21$ males, 3 females) and location-matched diabetic and nondiabetic FS ( $n=12$, mean age \pm SD $=58 \pm 14.9,7$ males, 5 females). Patient demographics and sample characteristics are included in Supplemental Table 1. DFU specimens were collected from discarded tissue after surgical debridement at the initial visit (W0) and 4 weeks after standard wound care (W4). Wounds were measured by planimetry, and patients were grouped as healing if there was more than $50 \%$ reduction or as nonhealing if there was less than $50 \%$ reduction at W4 compared with WO (59). Specimens were fixed in formalin and processed for paraffin embedding and stained with $\mathrm{H} \& \mathrm{E}$ to assess tissue morphology, as previously described (60).

FISH-Flow P-2 RNA assay and flow cytometric analysis. Single-cell suspensions were obtained from full-thickness DFU tissue and control skin as described before (23). Cells were first labeled with a live/ dead detection kit followed by labeling with fluorescently labeled antibodies CD45-Alexa Fluor 700 (BioLegend, 368514, clone 2D1), CD31-PacBlue (BioLegend, 303114, clone WM59), and CD104-FITC (BioLegend, 327806, clone 58XB4). P-2 mRNA was detected using an amplified signal FISH technique (PrimeFlow; Affymetrix/eBioscience, Thermo Fisher Scientific). For mRNA detection, target probe hybridization was performed using type 1 (Alexa Fluor 647) probes for P-2 as previously described (23). Approximately 20,000 cell events were acquired from each sample on a flow cytometer equipped with $405 \mathrm{~nm}$, $488 \mathrm{~nm}, 642 \mathrm{~nm}$, and $785 \mathrm{~nm}$ (SSC) lasers (Fortessa, BD Biosciences). Spectral compensation was completed using single-color control samples. Live $\mathrm{CD}^{-} 5^{-}, \mathrm{CD}^{-} 1^{-}$, and $\mathrm{CD} 104^{+}$cells were analyzed for expression of P-2 mRNA using FlowJo software (Tree Star version 10).

RNA isolation, $q P C R$, and genomic analyses. Total RNA was extracted using the miRNeasy kit (QIAGEN) per the manufacturer's instructions. cDNA was made with qScript Synthesis kit (Quanta BioSciences). P-2 qPCR reactions were performed in triplicate using PerfeCTa SYBR Green SuperMix (Quanta BioSciences) and P-2-specific primers (forward 5'-AAGGACCAAGCTATAACTACCCG-3; reverse 5'-CGGTCAGAGATGTCAAGGAGTTC- $3^{\prime}$ ), and quantified using the $\triangle \triangle \mathrm{CT}$ method. ARPC2 (forward 5'-TCCGGGACTACCTGCACTAC-3'; reverse 5'-GGTTCAGCACCTTGAGGAAG-3') was used as a reference gene 
for normalization. The microarray data set (deposited in NCBI's Gene Expression Omnibus under accession number GSE80178) was previously described (8). Raw data (.CEL) files were GCRMA normalized and filtered using GeneSpring 13.0, and a list of differentially expressed probes/corresponding genes was determined using a moderated $t$ test, $P$ less than 0.05 , fold change greater than 2 .

Isolation of intracellular bacteria. Full-thickness skin samples were minced and incubated at $37^{\circ} \mathrm{C}$ for 3 hours with $2 \mathrm{mg} / \mathrm{mL}$ collagenase $\mathrm{D}$ (Roche) at $37^{\circ} \mathrm{C}$ under constant agitation in the presence of $100 \mu \mathrm{g} /$ $\mathrm{mL}$ gentamycin, $25 \mu \mathrm{g} / \mathrm{mL}$ lysostaphin, and $100 \mu \mathrm{g} / \mathrm{mL}$ lysozyme (23). Obtained cell suspensions were washed with DMEM (Gibco, Thermo Fisher Scientific) supplemented with 10\% heat-inactivated FBS, 2 mM L-glutamine, $0.15 \%$ sodium hydrogen carbonate, $1 \mathrm{mM}$ sodium pyruvate, nonessential amino acids, $50 \mu \mathrm{g} / \mathrm{mL}$ gentamycin, $25 \mu \mathrm{g} / \mathrm{mL}$ lysostaphin, and $100 \mu \mathrm{g} / \mathrm{mL}$ lysozyme. To separate the epidermis and dermis, skin samples were cut in 1-2 mm strips and incubated overnight with Dispase II $(2.4 \mathrm{U} / \mathrm{mL})$ at $4^{\circ} \mathrm{C}$ in the presence of $100 \mu \mathrm{g} / \mathrm{mL}$ gentamycin, $25 \mu \mathrm{g} / \mathrm{mL}$ lysostaphin, and $100 \mu \mathrm{g} / \mathrm{mL}$ lysozyme. Epidermal cells were obtained by physical dissociation of epidermal sheets and vigorous pipetting. Dermis was additionally incubated for 1 hour with $2 \mathrm{mg} / \mathrm{mL}$ collagenase $\mathrm{D}$ (Roche) at $37^{\circ} \mathrm{C}$. The cells were pelleted, washed with PBS, and subsequently lysed with $100 \mu \mathrm{L}$ of $0.2 \%$ saponin in $1 \times$ PBS on ice for 20 minutes. Serial dilutions were plated on mannitol salt agar for bacterial quantification. Standard identification methods and PCR detection of the S. aureus-specific DNA gyrase A (gyrA) and staphylococcal nuclease (nuc) gene were used to identify $S$. aureus DFU isolates as described $(8,61)$.

Bacterial strains and growth conditions. MRSA USA300-0114 (62), MRSA USA300 JE2 (63), and $S$. aureus intracellular isolate from DFUs were used. MRSA USA300 AH1726 (MRSA LAC [AH1263] + pCM29 [CmR]) $(46,64)$ was used for murine in vivo skin infection. Todd-Hewitt broth supplemented with $0.2 \%$ yeast extract (THY) served as growth medium; oxacillin resistance screening agar base (OXOID) was used as selective media for MRSA CFU quantification.

Intracellular infection assay. Primary human keratinocytes were grown to approximately $50 \%$ confluency and infected with MRSA USA300-0114 or S. aureus isolate from the DFUs at an MOI of 100 (20). Cells were infected with MRSA at MOI of 100 for 15, 30, and 60 minutes; washed 3 times with PBS; and collected at 15-minute time increments to allow for bacterial adhesion to keratinocytes. In addition, primary human keratinocytes were infected for 60, 120, and 180 minutes; washed 3 times with sterile PBS to further remove any remaining extracellular bacteria; switched to $25 \mu \mathrm{g} / \mathrm{mL}$ lysostaphin to kill remaining extracellular bacteria; and collected at 30,60, and 120 minutes to allow for intracellular infection (20). Cells were harvested at indicated time increments for the ASC pyroptosome assay, immunofluorescence staining, and confocal imaging.

Mouse skin infection assay. WT mice (C57BL/6J $[n=7])$ were obtained from The Jackson Laboratory. P-2-KO mice $(n=9)$ were generated and bred at University of Miami $(20,65)$. Mice were shaved and tape-stripped (7 applications) with Transpore tape (3M) to disrupt the epidermal barrier (20). An inoculum of $10^{9}$ MRSA USA300 AH1726 in $0.02 \mathrm{~mL}$ of PBS or PBS control was added to approximately $1 \mathrm{~cm}^{2}$ of skin and the area bandaged with plastic sheet and overwrapped with dressings of Transpore tape and Nexcare waterproof tape (3M) for 24 hours. Upon 24 hours of infection, mice were euthanized and skin processed for protein isolation and CFU enumeration, followed by cryopreservation for imaging.
ASC pyroptosome assay. Tissue or cells were homogenized using $20 \mathrm{mM}$ HEPES, pH 7.5; $150 \mathrm{mM} \mathrm{KCl}$; 1\% NP-40; and 1× protease and phosphatase inhibitors (Cell Signaling Technology). Lysates were centrifuged at $304 g$ for 5 minutes and supernatant was collected. An equal amount of protein (50-100 $\mu \mathrm{g}$ ) was used to cross-link the ASC pyroptosome with $2 \mathrm{mM}$ disuccinimidyl suberate for 30 minutes. Pyroptosome was pelleted by centrifugation at $3381 \mathrm{~g}$ for 10 minutes. Pellet was resuspended with $2 \times$ SDS sample buffer and subjected to electrophoresis and immunoblot analysis with anti-ASC, as described below.

Western blot. Protein from each sample was resolved on 4\%-20\% Criterion TGX precast gels (Bio-Rad) and transferred onto PVDF membranes (Bio-Rad). Membranes were blocked with I-Block (Applied Biosystems) in PBS containing 0.1\% Tween 20, and then probed with anti-ASC (Novus Biologicals, NBP1-78977, 1:1000 and Cell Signaling Technology, 13833S, 1:500), human anti-AIM2 (Cell Signaling Technology, D5X7K, 1:1000), mouse anti-AIM2 (Abcam, AB93015, 1:1000), anti-caspase-1 (Cell Signaling Technology, 2225S, 1:1000), anti-gasdermin D (Novus Biologicals, NBP2-33422, 1:1000), or anti-caspase-3 (Cell Signaling Technology, 9662S, 1:1000). After washing, membranes were incubated with their appropriate HRP-conjugated secondary antibodies (Cell Signaling Technology, 7074S, 1:10,000 and 7076S, 1:10,000) and developed using an ECL Prime chemiluminescence detection system according to the instructions of the manufacturer (GE Healthcare). Immunoblots were stripped with Restore Plus Western blot stripping buffer (Pierce). Anti- $\beta$-actin antibody (Sigma-Aldrich, AS411, 1:50,000) was used for loading control.

Immunofluorescence staining. Paraffin-embedded tissue sections of DFUs were used for staining with anti-S. aureus (Abcam, ab20920, 1:100) as previously described (62). S. aureus was visualized with Alexa Fluor 488-conjugated goat anti-rabbit antibody (Invitrogen, A11008, 1:1000) and mounted with Prolong DAPI Gold antifade reagent (Invitrogen) to visualize cell nuclei. K17 (gift from P. Coulombe, Johns Hopkins University, Baltimore, Maryland, USA) was used for staining of keratinocytes in DFUs (66). For primary human keratinocyte staining, cells were fixed with $4 \%$ paraformaldehyde for 10 minutes at room temperature after infection with $S$. aureus as described above, followed by $3 \times$ washes with PBS. Cells were then permeabilized with $0.5 \%$ Triton $\mathrm{X}-100$ for 5 minutes and washed $3 \times$ with PBS. Cells were then stained with anti-S. aureus (Abcam, ab37644, 1:500) for 1 hour. S. aureus staining was visualized with Alexa Fluor 488-conjugated goat anti-mouse antibody (Invitrogen, A11029, 1:500) and mounted with Prolong DAPI Gold antifade reagent (Invitrogen) to visualize cell nuclei. Cells were then incubated with $200 \mathrm{nM}$ Acti-stain 555 phalloidin (Cytoskeletal, PHDR1) for 30 minutes followed by $3 \times$ PBS washes. Cryosectioned infected and control mouse skin embedded in OCT was fixed in $4 \%$ paraformaldehyde for 15 minutes at room temperature, rinsed in PBS, and permeabilized in $0.5 \%$ Triton X-100-PBS for 20 minutes. Slides were rinsed twice in PBS, blocked in 5\% BSA in PBS with 0.1\% Tween 20 (PBST) for 1 hour, and labeled with rabbit anti-S. aureus (Abcam, ab20920, 1:500) for 1 hour at room temperature. After 3 rinses in PBST, slides were fluorescently labeled with Alexa Fluor 488-conjugated goat anti-rabbit antibody (Invitrogen, A11008, 1:1000) for 1 hour. The samples were then incubated overnight at $4^{\circ} \mathrm{C}$ with rhodamine-conjugated phalloidin (200 nM, Cytoskeleton, PHDR1) and mounted with Prolong Diamond antifade reagent with DAPI (Invitrogen) to visualize the cellular nuclei. Images were obtained with an LSM 800 confocal microscope (Carl Zeiss $\mathrm{GmbH}$ ) in epifluorescence mode for lower resolution images 
and in laser scanning mode for higher resolution images. Image analysis was performed using Zeiss Zen 2.5 (blue edition) version 2.5.75.00006.

DNA extraction from FFPE DFU tissue and quantification of S. aureus by PCR. Total DNA from DFU FFPE blocks was extracted from $3 \mathrm{sec}-$ tions (10 $\mu \mathrm{m}$ thick) using the GeneRead DNA FFPE kit (QIAGEN) and qPCR quantification of $S$. aureus performed as described (8). The following primers were used: Nuc-F-162 GTTGTAGTTTCAAGTCTAAGTAGCTC, Nuc-R-162 AACCGTATCACCATCAATC, and Nucprobe-162 ATCCAACAGTATATAGTGCAAC (43). To measure microbial load, qPCR assays of the 16S rRNA gene were performed as previously described (9).

ELISA. Active IL-1 $\beta$ from DFUs was assessed by ELISA using the

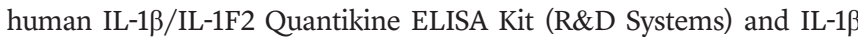
from murine skin using mouse IL-1B/IL-1F2 Quantikine ELISA Kit (R\&D Systems) according to the manufacturer's instructions. Samples were measured in duplicates and mean optical density values were used to normalize to total protein.

Statistics. The numbers of healthy FS and DFU samples, normal or P-2-KO mice, ELISA replicates, or cell replicates are indicated in each figure. All data are presented as mean \pm SD. Two-tailed Student's $t$ test and either 1-way or 2-way ANOVA were performed. $P$ values of less than 0.05 were considered significant. Cultured cell experiments were repeated at least 3 times and similar results were obtained.

Study approval. Ulcer tissue specimens were obtained after IRB approval (UM protocol 20150222), and written informed consent was obtained from participants prior to study participation. All animal experiments were performed in accordance with University of Miami IACUC guidelines under IACUC-approved protocol 16-006.

\section{Author contributions}

MTC, IP, APS, and NS designed research; IP, APS, JM, JLB, RCS, IJ, KER, TCW, CRH, OS, and EYK performed research; RSK, HLT, and NS contributed reagents; IP, APS, JM, JLB, NS, RCS, IJ, and MTC analyzed data; and IP, APS, and MTC wrote the manuscript.

\section{Acknowledgments}

This work was supported by NIH R01NR015649 (to MTC and NS), NIH U01DK119085-02S1 (to RSK, RCS, and MTC), and Frost Endowed Investigator fund (to MTC). We dedicate this work to the late Eckhard Podack, without whom studies of P-2 would not be possible. We are grateful to E. Thomas, G. Plano, A. Barrientos, R.W. Keane, and J.P. Vaccari for generously sharing laboratory resources and equipment. We also thank Alan Burgess and Ashley Rosa for technical support and our lab members for their continuous support and constructive criticism.

Address correspondence to: Irena Pastar, Wound Healing and Regenerative Medicine Research Program, Dr. Phillip Frost Department of Dermatology and Cutaneous Surgery, University of Miami Miller School of Medicine, 1600 NW 10th Avenue, RMSB, Room 6056, Miami, Florida 33136, USA. Phone: 305.689.7032; Email: IPastar@ med.miami.edu. Or to: Marjana Tomic-Canic, Wound Healing and Regenerative Medicine Research Program, Dr. Phillip Frost Department of Dermatology and Cutaneous Surgery, University of Miami Miller School of Medicine, 1600 NW 10th Avenue, RMSB, Room 2023A, Miami, Florida 33136, USA. Phone: 305.243.7295; Email: mtcanic@med.miami.edu.
1. Armstrong DG, et al. Diabetic foot ulcers and their recurrence. $N$ Engl JMed. 2017;376(24):2367-2375.

2. Singh N, et al. Preventing foot ulcers in patients with diabetes. JAMA. 2005;293(2):217-228.

3. Brem H, Tomic-Canic M. Cellular and molecular basis of wound healing in diabetes. JClin Invest. 2007;117(5):1219-1222.

4. Reiber GE. The epidemiology of diabetic foot problems. Diabet Med.1996;13(suppl 1):S6-S11.

5. Ghanassia E, et al. Long-term outcome and disability of diabetic patients hospitalized for diabetic foot ulcers: a 6.5-year follow-up study. Diabetes Care. 2008;31(7):1288-1292.

6. Holman N, et al. Variation in the recorded incidence of amputation of the lower limb in England. Diabetologia. 2012;55(7):1919-1925.

7. Kalan LR, et al. Strain- and species-level variation in the microbiome of diabetic wounds is associated with clinical outcomes and therapeutic efficacy. Cell Host Microbe. 2019;25(5):641-655.

8. Ramirez HA, et al. Staphylococcus aureus triggers induction of miR-15B-5P to diminish DNA repair and deregulate inflammatory response in diabetic foot ulcers. J Invest Dermatol. 2018;138(5):1187-1196.

9. Gardner SE, et al. The neuropathic diabetic foot ulcer microbiome is associated with clinical factors. Diabetes. 2013;62(3):923-930.

10. van Asten SAV, et al. The microbiome of diabetic foot osteomyelitis. Eur J Clin Microbiol Infect Dis. 2016;35(2):293-298.
11. Wolcott RD, et al. Analysis of the chronic wound microbiota of 2,963 patients by $16 \mathrm{~S}$ rDNA pyrosequencing. Wound Repair Regen. 2016;24(1):163-174.

12. Loesche $\mathrm{M}$, et al. Temporal stability in chronic wound microbiota is associated with poor heal ing. J Invest Dermatol. 2017;137(1):237-244.

13. Blakytny R, Jude EB. Altered molecular mechanisms of diabetic foot ulcers. Int J Low Extrem Wounds. 2009;8(2):95-104.

14. Dinh T, et al. Mechanisms involved in the development and healing of diabetic foot ulceration. Diabetes. 2012;61(11):2937-2947.

15. Fadini GP, et al. NETosis delays diabetic wound healing in mice and humans. Diabetes. 2016;65(4):1061-1071.

16. Eming SA, et al. Wound repair and regeneration: mechanisms, signaling, and translation. Sci Transl Med. 2014;6(265):265sr266.

17. Sawaya AP, et al. Deregulated immune cell recruitment orchestrated by FOXM1 impairs human diabetic wound healing. Nat Commun . 2020;11(1):4678.

18. McCormack R, et al. Killing machines: three pore-forming proteins of the immune system. Immunol Res. 2013;57(1-3):268-278.

19. McCormack R, et al. Inhibition of intracellular bacterial replication in fibroblasts is dependent on the perforin-like protein (perforin-2) encoded by macrophage-expressed gene 1. JInnate Immun. 2013;5(2):185-194.

20. McCormack RM, et al. Perforin-2 is essential for intracellular defense of parenchymal cells and phagocytes against pathogenic bacteria. Elife. 2015;4:e06508.

21. McCormack RM, et al. Enteric pathogens deploy cell cycle inhibiting factors to block the bactericidal activity of perforin-2. Elife. 2015;4:e06505.

22. McCormack RM, et al. MPEG1/perforin-2 mutations in human pulmonary nontuberculous mycobacterial infections. JCI Insight. 2017;2(8):e89635.

23. Strbo N, et al. Single cell analyses reveal specific distribution of anti-bacterial molecule perforin-2 in human skin and its modulation by wounding and Staphylococcus aureus infection. Exp Dermatol. 2019;28(3):225-232.

24. Spilsbury K, et al. Isolation of a novel macrophage-specific gene by differential cDNA analysis. Blood. 1995;85(6):1620-1629.

25. Soong G, et al. Methicillin-resistant Staphylococcus aureus adaptation to human keratinocytes. mBio. 2015;6(2):e00289-15.

26. Bergsbaken T, et al. Pyroptosis: host cell death and inflammation. Nat Rev Microbiol. 2009;7(2):99-109.

27. Fink SL, Cookson BT. Caspase-1-dependent pore formation during pyroptosis leads to osmotic lysis of infected host macrophages. Cell Microbiol. 2006;8(11):1812-1825.

28. Fernandes-Alnemri T, et al. The pyroptosome: a supramolecular assembly of ASC dimers mediating inflammatory cell death via caspase-1 activation. Cell Death Differ. 2007;14(9):1590-1604. 
29. Munoz-Arias I, et al. Blood-derived CD4 T cells naturally resist pyroptosis during abortive HIV-1 infection. Cell Host Microbe. 2015;18(4):463-470.

30. Bergsbaken T, Cookson BT. Macrophage activation redirects yersinia-infected host cell death from apoptosis to caspase-1-dependent pyroptosis. PLoS Pathog. 2007;3(11):e161.

31. Brennan MA, Cookson BT. Salmonella induces macrophage death by caspase-1-dependent necrosis. Mol Microbiol. 2000;38(1):31-40.

32. Rathinam VAK, et al. The AIM2 inflammasome is essential for host defense against cytosolic bacteria and DNA viruses. Nat Immunol. 2010;11(5):395-402.

33. Dombrowski Y, et al. Cytosolic DNA triggers inflammasome activation in keratinocytes in psoriatic lesions. Sci Transl Med. 2011;3(82):82ra38.

34. Fernandes-Alnemri T, et al. AIM2 activates the inflammasome and cell death in response to cytoplasmic DNA. Nature. 2009;458(7237):509-513.

35. Hornung V, et al. AIM2 recognizes cytosolic dsDNA and forms a caspase-1-activating inflammasome with ASC. Nature. 2009;458(7237):514-518.

36. Adamczak SE, et al. Pyroptotic neuronal cell death mediated by the AIM2 inflammasome. JCereb Blood Flow Metab. 2014;34(4):621-629.

37. Liu X, et al. Inflammasome-activated gasdermin D causes pyroptosis by forming membrane pores. Nature. 2016;535(7610):153-158.

38. Kang R, et al. Lipid peroxidation drives gasdermin D-mediated pyroptosis in lethal polymicrobial sepsis. Cell Host Microbe. 2018;24(1):97-108.

39. Contassot E, et al. Interleukin-1, inflammasomes, autoinflammation and the skin. Swiss Med Wkly. 2012;142:w13590.

40. de Koning HD, et al. Strong induction of AIM2 expression in human epidermis in acute and chronic inflammatory skin conditions. Exp Dermatol. 2012;21(12):961-964.

41. Merselis LC, et al. MPEG1/perforin-2 haploinsufficiency associated polymicrobial skin infections and considerations for interferon- $\gamma$ therapy. Front Immunol. 2020;11:601584.

42. Margolis DJ, et al. Surrogate end points for the treatment of diabetic neuropathic foot ulcers.
Diabetes Care. 2003;26(6):1696-1700.

43. Redel H, et al. Quantitation and composition of cutaneous microbiota in diabetic and nondiabetic men. JInfect Dis. 2013;207(7):1105-1114.

44. Ramirez HA, et al. Comparative genomic, microRNA, and tissue analyses reveal subtle differences between non-diabetic and diabetic foot skin. PLoS One. 2015;10(8):e0137133.

45. Podack ER, Munson GP. Killing of microbes and cancer by the immune system with three mammalian pore-forming killer proteins. Front Immunol. 2016;7:464.

46. Pastar I, et al. Staphylococcus epidermidis boosts innate immune response by activation of gamma delta T cells and induction of perforin- 2 in human skin. Front Immunol. 2020;11:550946.

47. Nassiri S, et al. Relative expression of proinflammatory and antiinflammatory genes reveals differences between healing and nonhealing human chronic diabetic foot ulcers. J Invest Dermatol. 2015;135(6):1700-1703.

48. Mirza RE, et al. Blocking interleukin-1 $\beta$ induces a healing-associated wound macrophage phenotype and improves healing in type 2 diabetes. Diabetes. 2013;62(7):2579-2587.

49. Mast BA, Schultz GS. Interactions of cytokines, growth factors, and proteases in acute and chronic wounds. Wound Repair Regen. 1996;4(4):411-420.

50. Fivenson DP, et al. Chemokine and inflammatory cytokine changes during chronic wound healing. Wound Repair Regen. 1997;5(4):310-322.

51. Tarnuzzer RW, Schultz GS. Biochemical analysis of acute and chronic wound environments. Wound Repair Regen. 1996;4(3):321-325.

52. Stojadinovic O, et al. Stress-induced hormones cortisol and epinephrine impair wound epithelization. Adv Wound Care (New Rochelle). 2012;1(1):29-35.

53. Vukelic $S$, et al. Cortisol synthesis in epidermis is induced by IL-1 and tissue injury. J Biol Chem. 2011;286(12):10265-10275.

54. Jozic I, et al. Glucocorticoid-mediated induction of caveolin-1 disrupts cytoskeletal organization, inhibits cell migration and re-epithelialization of non-healing wounds. Commun Biol. 2021;4(1):757.
55. Darby IA, et al. Apoptosis is increased in a model of diabetes-impaired wound healing in genetically diabetic mice. Int J Biochem Cell Biol. 1997;29(1):191-200.

56. Khanna S, et al. Macrophage dysfunction impairs resolution of inflammation in the wounds of diabetic mice. PLoS One. 2010;5(3):e9539.

57. Lewis CJ, et al. Bone morphogenetic protein signaling suppresses wound-induced skin repair by inhibiting keratinocyte proliferation and migration. J Invest Dermatol. 2014;134(3):827-837.

58 . Tsuchiya K, et al. Caspase-1 initiates apoptosis in the absence of gasdermin D. Nat Commun. 2019;10(1):2091.

59. Sheehan P, et al. Percent change in wound area of diabetic foot ulcers over a 4 -week period is a robust predictor of complete healing in a 12-week prospective trial. Diabetes Care. 2003;26(6):1879-1882.

60. Glinos GD, et al. Optical coherence tomography for assessment of epithelialization in a human ex vivo wound model. Wound Repair Regen. 2017;25(6):1017-1026.

61. Rolle CE, et al. Keratinocytes produce IL-6 in response to desmoglein 1 cleavage by Staphylococcus aureus exfoliative toxin A. Immunol Res. 2013;57(1-3):258-267.

62. Pastar I, et al. Interactions of methicillin resistant Staphylococcus aureus USA300 and Pseudomonas aeruginosa in polymicrobial wound infection. PLoS One. 2013;8(2):e56846.

63. Fey PD, et al. A genetic resource for rapid and comprehensive phenotype screening of nonessential Staphylococcus aureus genes. mBio. 2013;4(1):e00537-e00512.

64. Pang YY, et al. agr-dependent interactions of Staphylococcus aureus USA300 with human polymorphonuclear neutrophils. JInnate Immun. 2010;2(6):546-559.

65. Gayle P, et al. Maternal- and fetal-encoded perforin-2 limits placental infection by a bloodborne pathogen. JImmunol. 2020;205(7):1878-1885.

66. McGowan KM, Coulombe PA. Onset of keratin 17 expression coincides with the definition of major epithelial lineages during skin development. JCell Biol. 1998;143(2):469-486. 